\title{
Filtermaterialprüfung: Anwendung der ÖNORM B 2506 Teil 3 für das hochrangige Straßennetz
}

\author{
Tadele Measho Haile · Maria Fürhacker
}

Online publiziert: 18. September 2017

(c) Der/die Autor(en) 2017. Dieser Artikel ist eine Open-Access-Publikation.

\begin{abstract}
Zusammenfassung Verkehrsflächenabflüsse können mit organischen und anorganischen Stoffen belastet sein und als verunreinigt gelten, sodass sie vor Einbringung in den Untergrund gereinigt werden müssen. Die Belastungen stammen bzw. entstehen aus Abgasnebenprodukten, Reifen-, Karosserieund Fahrbahnverschleiß, Abflüssen aus Niederschlägen, nasser und trockener Deposition und Fahrbahninstandhaltungsarbeiten. Im ÖWAV-Regelblatt 45 und in der ÖNORM B 2506, Teil 1 und 2 wird der Stand der Technik der Reinigung vor Versickerung in den Un-

dass die Prüfung der technischen Filtermaterialien nach ÖNORM B 2506-3 für die Versickerung in das Grundwasser erstellt wurde.

Schlüsselwörter Straßenabwässer . Materialprüfung · ÖNORM B 2506 Teil 3 - Hochrangige Straßen · JDTV $>15.000$

\section{Testing methods for}

filtermaterials: Application of ÖNORM B 2506 Part 3 for roads with high annaual average daily traffic (AADT)
\end{abstract} tergrund mit Bodenfiltern bzw. „technischen Bodenfiltern" (ÖNORM) und „technischen Filtern“ (ÖWAV-RB 45) beschrieben. Die Kriterien der Mindestleistungsfähigkeit und deren Prüfung wurden in der ÖNORM B 2506, Teil 3 festgelegt. Da sowohl in der ÖNORM B 2506, Teil 1 und 2 als auch im ÖWAVRB 45 die hochrangigen Straßen ausgenommen wurden, sollen in diesem Artikel die Grundlagen der ÖNORM B 2506, Teil 3 erläutert und ihre Anwendbarkeit auch auf hochrangige Straßen aufgezeigt werden. Es konnte gezeigt werden, dass aufgrund der in der ÖNORM B 2506-3 gewählten strengen Prüfbedingungen und Prüfkriterien die Prüfung der technischen Filtermaterialien aus wissenschaftlicher Sicht geeignet sind, auch die Anforderungen an die Reinigung von Straßenabwässern von hochbelasteten Straßen mit hohen durchschnittlichen täglichen Verkehrsbelastungen (JDTV), wie jenen des hochrangigen Straßennetzes, zu erfüllen. Es sei noch darauf hingewiesen,

\section{T. M. Haile}

Univ.-Prof. DI Dr. M. Fürhacker $(\bowtie)$

Department für Wasser,

Atmosphäre und Umwelt,

Institut für Siedlungswasserbau, Industriewasserwirtschaft und

Gewässerschutz, Universität

für Bodenkultur Wien,

Muthgasse 18, 1190 Wien, Österreich

maria.fuerhacker@boku.ac.at
Abstract Street runoff can be contaminated with organic and inorganic substances, and therefore have to be treated before being infiltrated in the underground. The contaminations are from tires, vehicles, roads, precipitation, wet and dry deposition, road maintenance work or are generated by exhaust gas products. The state of the art of cleaning before infiltration into the underground is described in the ÖWAV-Regelblatt 45 and ÖNORM B 2506 Parts 1 \& 2, with the help of soil filters or "technical soil filters" (ÖNORM) and "technical filters" (ÖWAV-RB 45). The testing methods and performance criteria for such filters have been defined in ÖNORM B 2506 Part 3. Since both the ÖNORM B 2506 Parts 1 \& 2 and the ÖWAV-RB 45 have exempted the high-ranking roads, this article explains the background of ÖNORM B2506 Part 3 and its applicability for run-off treatment of highranking roads. It has been shown that due to the strict test conditions and test criteria chosen in ÖNORM B 2506-3, the testing of the technical filter materials is from a scientific point of view also suitable, to meet the requirements for roads with high annual average daily traffic (AADT). It should be pointed out that the testing methods according to ÖNORM B 2506-3 was designed for infiltration into the underground.

\section{Einleitung}

Abwässer von Straßen können relativ stark mit Spurenstoffen belastet sein. In der Qualitätszielverordnung Chemie Grundwasser ist ein Verbot der direkten Einbringung von Schadstoffen in das Grundwasser enthalten, wobei unter direkter Einbringung jede dauernde oder zeitweilige Einbringung von Schadstoffen in das Grundwasser ohne Bodenpassage zu verstehen ist. Aus diesem Grund ist die Behandlung von Straßenabwässern besonders bei stark befahrenen Straßen vor der Einleitung in ein Gewässer oder in den Untergrund erforderlich. Im ÖWAV-Regelblatt 45 (ÖWAV-RB 45 2015) und in der ÖNORM B 2506, Teil 1 und 2 wird der Stand der Technik der Reinigung vor Versickerung in den Untergrund beschrieben. Da in den Vorschriften neben Bodenfiltern auch Aussagen zu ,technischen Bodenfiltern" (ÖNORM B 2506-2 2012) bzw. $\mathrm{zu}$ „technischen Filtern“ (ÖWAV-RB 45 2015) gemacht werden, war es notwendig, diese zu charakterisieren bzw. eine Mindestleistungsfähigkeit $\mathrm{zu}$ fordern. Die Kriterien der Mindestleistungsfähigkeit und deren Prüfung wurden in der ÖNORM B 2506-Teil 3 (ÖNORM B 2506-3 2016) festgelegt.

Allerdings sind sowohl in der ÖNORM B 2506-Teil 3 als auch im ÖWAV-RB 45 die hochrangigen Straßen nicht berücksichtigt, weil diese durch die Vorschriften der FSG in den RVS konkretisiert wurden. Ziel dieses Artikels ist es, die Grundlagen der ÖNORM B 2506-Teil 3 zu erläutern und ihre Anwendbarkeit auch auf hochrangige Straßen aufzuzeigen.

\section{Verunreinigungen von Straßenabwässern}

Verkehrsflächenabflüsse können mit organischen und anorganischen Spurenstoffen, Feststoffen (z. B. abfiltrierbaren Stoffen) und Nährstoffen belastet sein (Fürhacker et al. 2013; Geiger-Kaiser und Jäger 2005; Göbel et al. 2007; 


\begin{tabular}{|c|c|}
\hline Stoffe & Hauptquellen \\
\hline Ba & Bremsbeläge und Verkleidungen \\
\hline $\mathrm{Cd}$ & Reifenverschleiß, Bremsbeläge, Schmieröle, Verbrennung und Korrosion \\
\hline Co & Verschleiß von Spikereifen, Korrosion von Buchsen, Bremsdrähten und Heizkörpern \\
\hline $\mathrm{Cr}$ & Bewegliche Motorenteile, Bremsbeläge, Asphalt- und Fahrbahnverschleiß, Korrosion von geschweißten Metallbeschichtungen und Lacken \\
\hline $\mathrm{Cu}$ & Lager- und Buchsenverschleiß, bewegliche Motorteile, Bremsbeläge, Reifenverschleiß, Asphalt- und Fahrbahnverschleiß und Schmieröle \\
\hline $\mathrm{Ni}$ & $\begin{array}{l}\text { Automobil-Emission, Schmieröl, Korrosion von Karosserieteilen, Bremsbeläge, Asphalt und Fahrbahnverschleiß und Verschleiß von beweglichen } \\
\text { Teilen in Motoren, Katalysatoren }\end{array}$ \\
\hline $\mathrm{Pb}$ & $\begin{array}{l}\text { Fahrzeugabgase, Reifenverschleiß, Schmieröle, Fett, Bremsbeläge, Lagerverschleiß, Asphalt- und Fahrbahnverschleiß sowie Verschleiß beweglicher } \\
\text { Teile in Motoren }\end{array}$ \\
\hline Pt & Katalysatoren \\
\hline $\mathrm{Sb}$ & Bremsbeläge und Verkleidungen \\
\hline $\mathrm{Sr}$ & Bremsbeläge und Verkleidungen \\
\hline Ti & Bremsbeläge und Verkleidungen \\
\hline V & Reifenverschleiß, Asphalt und Fahrbahnverschleiß \\
\hline $\mathrm{Zn}$ & Reifenverschleiß, Motoröl, Fett, Bremsbeläge, Asphalt- und Fahrbahnverschleiß und Schmieröle \\
\hline PAK & Reifenverschleiß, Fahrzeugabgase, Asphaltabnutzung/Straßenalterung \\
\hline MKW & Motorölaustritt, Fahrzeugabgase, Kraftstoffe und Frostschutzmittel, Verflüchtigungsverlust \\
\hline
\end{tabular}

Helmreich et al. 2010; Sansalone und Buchberger 1997). Die Belastung hängt im Wesentlichen von verschiedenen Parametern wie Witterungsbedingungen (Dauer von Trockenperioden und Niederschlagsintensität), Verkehrsfrequenz oder der jahresdurchschnittlichen täglichen Verkehrsbelastung (JDTV) und der Art der Nutzung (z. B. „stop-and go" im Kreuzungsbereich, Beschleunigungsspur, Parkfläche) ab. Diese unerwünschten Niederschlagsbestandteile stammen bzw. entstehen aus Abgasnebenprodukten, Reifen-, Karosserieund Fahrbahnverschleiß, Abflüssen aus Niederschlägen, nasser und trockener Deposition und Fahrbahninstandhaltungsarbeiten.

Die Spurenstoffe, die durch den Kraftfahrzeugverkehr in den Gewässerkreislauf eingetragen werden können, sind in Tab. 1 zusammengefasst.

\section{Konzentrationen verschiedener Kontaminationen}

Die Konzentrationen von Verunreinigungen wie ungelöste Stoffe, Schwermetalle und organische Verbindungen (z. B. MKW und PAK) in Niederschlagsabflüssen von hochrangigen Straßen können so hoch sein, dass diese als verschmutzt eingestuft werden und vor der Einleitung in Gewässer oder Grundwasser zu behandeln sind

Tab. 2 gibt einen Auszug aus Literaturdaten $\mathrm{zu}$ Schwermetallen, MKW und PAK in Verkehrsflächenabflüssen wieder. In Tab. 2 ist erkennbar, dass die Schwermetallkonzentrationen auch innerhalb vergleichbarer JDTV-Belastungen sehr unterschiedlich sind und z. B. für $\mathrm{Cu}$ im Bereich 25 bis $682 \mu \mathrm{g} / \mathrm{l}$ schwanken. Tendenziell sieht man eine geringere Konzentration bei kleineren JDTV. Für $\mathrm{Pb}$ liegen die älteren Werte im oberen Konzentrationsbereich $>50 \mu \mathrm{g} / \mathrm{l}$. In der Zusammenfassung von Huber et al. (2016), die nur Werte nach dem Jahr 2000 gelistet haben, liegt die mittlere $\mathrm{Pb}$-Konzentration bei $13 \mu \mathrm{g} / \mathrm{l}$.

Box-Whisker-Plots von der Konzentrationsmatrix aus Tab. 2 ist in Abb. 1 dargestellt. Die Ergebnisse zeigten dass der Verkehrsfrequenz (JDTV) keinen größtmöglichen Einfluss auf die Konzentrationen der Schadstoffe hatte. Die höchsten Gesamtgehalte an $\mathrm{Cu}$, Ni und $\mathrm{Zn}$ wurden in Autobahnabflüssen an der A23 in Wien, Österreich gemessen (Fürhacker et al. 2013). Sehr hohe Gesamt-Pb-Konzentrationen (200 bis $250 \mu \mathrm{g} / \mathrm{l}$ ) wurden in Autobahnabflüssen an der A23 in Wien, Österreich (Fürhacker et al. 2013), an der A81 (Pleidelsheim) und A6 (Obereisesheim), Deutschland (Stotz 1987), I-94, Minneapolis, USA (Thomson et al. 1997) und 31 Autobahnen in 11 Staaten, USA (Driscoll et al. 1990) gemessen. Die höchsten gesamten 16 EPA-PAKKonzentrationen in Autobahnabflüssen wurden in absteigender Reihenfolge an der M7 in Monasterevin bypass, Ireland
(Desta et al. 2007), an der A23 in Wien, Österreich (Fürhacker et al. 2013), und an der M7 in Kildare, Ireland (Desta et al. 2007) bestimmt (Abb. 1).

Die Spurenstoffkonzentrationen sind nicht nur von den JDTV abhängig. In einigen Studien konnte ein Zusammenhang zwischen Schwermetallbelastung in Autobahnabflüssen und JDTV hergestellt werden (Crabtree et al. 2008; Horstmeyer und Helmreich 2014). Anhand der Schwermetallbelastung von Versickerungsmulden fanden Horstmeyer und Helmreich (2014) einen Zusammenhang zwischen Schwermetallbelastung des Oberbodens und JDTV; über 80.000 JDTV erfolgte keine weitere Zunahme der Kontamination. Die Ergebnisse dieser Literaturstudie zeigen, dass das JDTV keinen signifikanten Einfluss auf die Schadstoffkonzentrationen hat. Ein höheres Verkehrsaufkommen (JDTV > 15.000) allein führt somit nicht zu einem Anstieg der Schadstoffkonzentrationen im Straßenabwasser. Auch andere Studien (Herrera 2007; Kayhanian et al. 2003, 2012) ermittelten eine ähnliche Schlussfolgerung.

Die Schadstoffkonzentrationen in Niederschlagsabflüssen von Verkehrsflächen zeigten ortsspezifisch signifikante Unterschiede. Diese Variabilität resultiert nicht nur aus der Verkehrsdichte, sondern auch aus den Unterschieden in der Landnutzung, des gesamten kumulativen Niederschlags, der vorangegangenen Trockenperioden 
Tab. 2 Schwermetalle, PAK und MKW-Straßenablaufkonzentrationen aus der Literatur. (Zusammengefasst von Haile und Fürhacker)

\begin{tabular}{|c|c|c|c|c|c|c|c|c|c|c|c|c|}
\hline \multirow[t]{2}{*}{ Land } & \multirow[t]{2}{*}{ Ort } & \multirow[t]{2}{*}{ JDTV } & \multirow[t]{2}{*}{$\begin{array}{l}\text { Untersuchungs- } \\
\text { zeitraum }\end{array}$} & \multirow[t]{2}{*}{$\begin{array}{l}\text { Probe- } \\
\text { anzahl }\end{array}$} & \multirow{2}{*}{$\begin{array}{l}\mathrm{Cr} \\
\mu \mathrm{g} / \mathrm{l}\end{array}$} & \multirow{2}{*}{$\begin{array}{l}\mathrm{Cu} \\
\mu \mathrm{g} / \mathrm{l}\end{array}$} & \multirow{2}{*}{$\begin{array}{l}\mathrm{Ni} \\
\mu \mathrm{g} / \mathrm{l}\end{array}$} & \multirow{2}{*}{$\begin{array}{l}\mathrm{Pb} \\
\mu \mathrm{g} / \mathrm{l}\end{array}$} & \multirow{2}{*}{$\begin{array}{l}\mathrm{Zn} \\
\mu \mathrm{g} / \mathrm{l}\end{array}$} & \multirow{2}{*}{$\begin{array}{l}\text { PAK } \\
\mathrm{mg} / \mathrm{l}\end{array}$} & \multirow{2}{*}{$\begin{array}{l}\text { MKW } \\
\text { mg/l }\end{array}$} & \multirow[t]{2}{*}{ Literatur } \\
\hline & & & & & & & & & & & & \\
\hline AUT & $\begin{array}{l}\text { Autobahn A23, } \\
\text { Wien }\end{array}$ & 255.000 & 23.03.2011 & 1 & 72 & 682 & 69 & 112 & 2560 & 18 & 3 & Fürhacker et al. (2013) \\
\hline AUT & $\begin{array}{l}\text { Autobahn A23, } \\
\text { Wien }\end{array}$ & 255.000 & 11.07 .2013 & 1 & 11 & 131 & 9,8 & 195 & 633 & - & - & Fürhacker et al. (2013) \\
\hline AUT & $\begin{array}{l}\text { Autobahn A21, } \\
\text { Hinterbrühl }\end{array}$ & 42.000 & $12.2005-05.2008$ & 10 & - & 205 & - & - & 360 & 3,01 & 2 & Fuerhacker et al. (2011) \\
\hline AUT & $\begin{array}{l}\text { Autobahn, Mönchs- } \\
\text { graben }\end{array}$ & 60.530 & - & - & 78 & 145 & 29 & 22 & 520 & - & - & Höfler et al. (2004) \\
\hline AUT & $\begin{array}{l}\text { Autobahn A1, } \\
\text { Schwarzenbergka- } \\
\text { serne, Salz }\end{array}$ & $>60.000$ & $12.2001-04.2003$ & 29 & $<1$ & 41 & 2 & 8 & 110 & - & 0,036 & $\begin{array}{l}\text { Geiger-Kaiser und Jäger } \\
\text { (2005) }\end{array}$ \\
\hline AUT & $\begin{array}{l}\text { Autobahn A1, Bau- } \\
\text { los West, Salzburg }\end{array}$ & $>60.000$ & $12.2001-04.2003$ & 29 & 3 & 43 & 3 & 7 & 88 & - & 0,194 & $\begin{array}{l}\text { Geiger-Kaiser und Jäger } \\
\text { (2005) }\end{array}$ \\
\hline AUT & $\begin{array}{l}\text { Autobahn A1, Bau- } \\
\text { los 0st, Salzburg }\end{array}$ & $>60.000$ & $12.2001-04.2003$ & 29 & 6 & 59 & 3 & 11 & 260 & - & 0,205 & $\begin{array}{l}\text { Geiger-Kaiser und Jäger } \\
\text { (2005) }\end{array}$ \\
\hline ATU & $\begin{array}{l}\text { Landesstraße L202 } \\
\text { Hard-Bregenz }\end{array}$ & 26.000 & $01.2005-06.2006$ & 9 & 14 & 61 & 11 & 9,8 & 204 & - & 0,7 & $\begin{array}{l}\text { Scheffknecht und Prodinger } \\
\text { (2007) }\end{array}$ \\
\hline ATU & Pilotuntersuchung & $\begin{array}{l}26.000- \\
60.500\end{array}$ & - & 9 & 10 & 67 & 4,6 & 14 & 302 & - & 2,06 & Clara et al. (2014) \\
\hline DEU & $\begin{array}{l}\text { Landshuter Alle, } \\
\text { München }\end{array}$ & 57.000 & $11.2003-11.2005$ & 57 & - & 191 & 43 & 56 & 847 & - & - & Helmreich et al. (2010) \\
\hline DEU & $\begin{array}{l}\text { Landshuter Alle, } \\
\text { München }\end{array}$ & 57.000 & $11.2003-11.2005$ & 350 & - & 194 & - & 37 & 933 & - & - & Hilliges et al. (2013) \\
\hline DEU & A3, Köln-0st & 156.000 & $11.2006-08.2007$ & 20 & 3 & 32 & 2,4 & 4,9 & 102 & 0,15 & 0,14 & $\begin{array}{l}\text { Grotehusmann und Kasting } \\
\text { (2009) }\end{array}$ \\
\hline DEU & A113, Berlin & 140.000 & $11.2006-08.2007$ & 20 & 5,9 & 26 & 2,5 & 4,9 & 115 & 0,28 & 0,04 & $\begin{array}{l}\text { Grotehusmann und Kasting } \\
\text { (2009) }\end{array}$ \\
\hline DEU & $\begin{array}{l}\text { Autobahn mit un- } \\
\text { terschiedlicher } \\
\text { DTV }\end{array}$ & $\begin{array}{l}52.000- \\
107.600\end{array}$ & 20 Woche (1997) & 20 & - & 140 & - & 17 & 1250 & - & - & Dierkes und Geiger (1999) \\
\hline DEU & A 555 Widdig & 69.368 & $2005-2006$ & 65 & 5 & 27 & 10 & 25 & 106 & - & - & Kocher et al. (2010) \\
\hline DEU & A 61 Meckenheim & 73.310 & 2005-2006 & 63 & 5 & 25 & 10 & 25 & 260 & - & - & Kocher et al. (2010) \\
\hline DEU & A4 Bensberg & 71.220 & 2005-2006 & 76 & 5 & 27 & 11 & 25 & 355 & - & - & Kocher et al. (2010) \\
\hline DEU & $\begin{array}{l}\text { Hauptstraße, Litera- } \\
\text { turstudie }\end{array}$ & $>15.000$ & - & - & 11 & 97 & 11 & 170 & 407 & 1,65 & 4,17 & Göbel et al. (2007) \\
\hline DEU & $\begin{array}{l}\text { Autobahn, Literatur- } \\
\text { studie }\end{array}$ & $>30.000$ & - & - & 11 & 36 & 15 & 13 & 217 & - & - & Huber et al. (2016) \\
\hline DEU & Pleidelsheim A81 & 41.000 & - & - & 9,6 & 97 & - & 200 & 360 & - & 7,02 & Stotz (1987) \\
\hline DEU & Obereisesheim A6 & 47.000 & - & - & 20 & 117 & - & 250 & 620 & - & 5,51 & Stotz (1987) \\
\hline DEU & Ulm-West A8 & 52.100 & - & - & 5,2 & 58 & - & 160 & 320 & - & 2,05 & Stotz (1987) \\
\hline DEU & Halenreie, Hamburg & 15.000 & $08.2008-07.2010$ & 8 & - & 130 & - & - & 400 & - & - & Dobner und Holthuis (2011) \\
\hline DEU & Berlin & $\begin{array}{l}15.000- \\
20.000\end{array}$ & - & 10 & - & 127 & 29 & 80 & 500 & - & - & Schütte (1997) \\
\hline $\mathrm{CH}$ & $\begin{array}{l}\text { N1, Winterthur, } \\
\text { Zürich }\end{array}$ & $\begin{array}{l}25.300- \\
73.700\end{array}$ & $08.1996-11.2000$ & $>10$ & - & 57 & - & 26 & 354 & - & - & Furumai et al. (2002) \\
\hline $\mathrm{CH}$ & SABA Attinghausen & 21.000 & $03.2007-12.2008$ & 17 & 13 & 60 & 6 & 9 & 346 & 2,7 & - & Steiner et al. (2008) \\
\hline $\mathrm{CH}$ & SABA Burgdorf & $>17.000$ & $08.2002-11.2004$ & - & 10 & 57 & 7 & 23 & 299 & 2,6 & 1,46 & Langbein et al. (2006) \\
\hline $\mathrm{CH}$ & $\begin{array}{l}\text { A1 Mattstetten, } \\
\text { Bern }\end{array}$ & 60.000 & 2006-2007 & - & 12 & 127 & 7 & 19 & 381 & 2,3 & - & Scheiwiller et al. (2008) \\
\hline $\mathrm{CH}$ & SABA Burgdorf & $>17.000$ & $01.2003-02.2004$ & $>20$ & 16 & 66 & 7 & 24 & 436 & - & - & Steiner et al. (2006) \\
\hline $\mathrm{CH}$ & $\begin{array}{l}\text { Basel-Landschaft, } \\
\text { Birsfelden, N2, } \\
\text { Hagnau }\end{array}$ & $>120.000$ & 2008-2012 & 65 & - & 57 & - & - & 188 & - & - & Zbinden et al. (2015) \\
\hline $\mathrm{CH}$ & $\begin{array}{l}\text { Stark befahrenen } \\
\text { Straßen, Literatur- } \\
\text { studie }\end{array}$ & $>15.000$ & - & - & 8,9 & 34 & 11 & 15 & 118 & - & 0,293 & Hürlimann (2011) \\
\hline
\end{tabular}




\begin{tabular}{|c|c|c|c|c|c|c|c|c|c|c|c|c|}
\hline \multirow[t]{2}{*}{ Land } & \multirow[t]{2}{*}{ Ort } & \multirow[t]{2}{*}{ JDTV } & \multirow[t]{2}{*}{$\begin{array}{l}\text { Untersuchungs- } \\
\text { zeitraum }\end{array}$} & \multirow[t]{2}{*}{$\begin{array}{l}\text { Probe- } \\
\text { anzahl }\end{array}$} & \multirow{2}{*}{$\begin{array}{l}\mathrm{Cr} \\
\mu \mathrm{g} / \mathrm{l}\end{array}$} & \multirow{2}{*}{$\begin{array}{l}\mathrm{Cu} \\
\mu \mathrm{g} / \mathrm{l}\end{array}$} & \multirow{2}{*}{$\begin{array}{l}\mathrm{Ni} \\
\mu \mathrm{g} / \mathrm{l}\end{array}$} & \multirow{2}{*}{$\begin{array}{l}\mathrm{Pb} \\
\mu \mathrm{g} / \mathrm{l}\end{array}$} & \multirow{2}{*}{$\begin{array}{l}\mathrm{Zn} \\
\mu \mathrm{g} / \mathrm{l}\end{array}$} & \multirow{2}{*}{$\begin{array}{l}\text { PAK } \\
\mathrm{mg} / \mathrm{l}\end{array}$} & \multirow{2}{*}{$\begin{array}{l}\text { MKW } \\
\text { mg/l }\end{array}$} & \multirow[t]{2}{*}{ Literatur } \\
\hline & & & & & & & & & & & & \\
\hline USA & Los Angeles, USA & $\begin{array}{l}260.000- \\
328.000\end{array}$ & 2002-2003 & 62 & 10 & 93 & 20 & 33 & 506 & 0,4 & - & Lau et al. (2009) \\
\hline USA & $\begin{array}{l}\text { Non-urban } \\
\text { highways (16), } \\
\text { Caifornia }\end{array}$ & $\begin{array}{l}2100- \\
29.000\end{array}$ & 2002-2003 & 635 & 6,5 & 12 & 11 & 17 & 75,9 & - & - & Kayhanian et al. (2007) \\
\hline USA & $\begin{array}{l}\text { Urban highways (8), } \\
\text { Caifornia }\end{array}$ & $\begin{array}{l}30.000- \\
100.000\end{array}$ & 2002-2003 & 635 & 6,4 & 27 & 7,8 & 24 & 134 & - & - & Kayhanian et al. (2007) \\
\hline USA & $\begin{array}{l}\text { Urban highways } \\
\text { (10), Caifornia }\end{array}$ & $\begin{array}{l}100.000- \\
328.000\end{array}$ & - & 635 & 12 & 50 & 13 & 75 & 261 & - & - & Kayhanian et al. (2007) \\
\hline USA & Ohio, USA & 150.000 & 04.1995-11.1995 & 5 & 21 & 135 & 43 & 64 & 470 & - & - & $\begin{array}{l}\text { Sansalone und Buchberger } \\
\text { (1997) }\end{array}$ \\
\hline USA & $\begin{array}{l}\text { Austin, Texas area } \\
\text { USA }\end{array}$ & 58.150 & 09.1993-05.1995 & - & - & 37 & 25 & 53 & 222 & - & - & Barrett et al. (1998) \\
\hline USA & $\begin{array}{l}31 \text { Autobahnen in } \\
11 \text { Staaten }\end{array}$ & $>30.000$ & - & - & - & 54 & - & 234 & 368 & - & - & Driscoll et al. (1990) \\
\hline USA & I-94, Minneapolis & 114.000 & - & 136 & 13 & 47 & 10 & 207 & 174 & - & - & Thomson et al. (1997) \\
\hline UK & $\begin{array}{l}\text { M4, Brinkworth } \\
\text { Brook }\end{array}$ & 71.930 & 12.1997-12.1998 & 10 & ND & 24 & ND & ND & 101 & - & - & Moy et al. (2003) \\
\hline UK & A417, River Frome & 23.650 & 06.1998-07.1999 & 10 & 12 & 55 & 12 & 51 & 222 & - & - & Moy et al. (2003) \\
\hline UK & M4, River Ray & 36.110 & $12.1998-03.2000$ & 10 & 9,1 & 68 & 6,7 & 51 & 220 & - & - & Moy et al. (2003) \\
\hline UK & $\begin{array}{l}\text { M40, Souldern } \\
\text { Brook }\end{array}$ & 83.580 & 08.1999-11.2000 & 10 & 7,7 & 32 & 4 & 17 & 98 & - & - & Moy et al. (2003) \\
\hline UK & A34, Gallos Brook & 64.950 & $09.2000-03.2002$ & 10 & 4,8 & 43 & 4,5 & 15 & 149 & - & - & Moy et al. (2003) \\
\hline UK & A34, Newbury & 37.190 & $05.2001-06.2002$ & 10 & 2,7 & 24 & 4,7 & 4,4 & 52,5 & 1,73 & - & Moy et al. (2003) \\
\hline UK & $\begin{array}{l}4 \text { Klimaregionen } \\
\text { (6 Autobahn/ } \\
\text { Region) }\end{array}$ & $\begin{array}{l}15.000- \\
>120.000\end{array}$ & $06.2004-12.2006$ & 240 & - & 91 & - & - & 353 & 7,5 & - & Crabtree et al. (2008) \\
\hline IRL & $\begin{array}{l}\text { Motorway, Kildare/ } \\
\text { Portlaoise }\end{array}$ & 32.000 & 08.2005-10.2005 & 6 & - & 46 & - & 67 & 181 & - & - & Gill et al. (2017) \\
\hline IRL & Kildare & 25.760 & 08.2003-12.2005 & 42 & - & 120 & - & 140 & 660 & 11 & - & Desta et al. (2007) \\
\hline IRL & Maynooth & 29.140 & 08.2003-12.2005 & 42 & - & - & - & - & 70 & 2,06 & - & Desta et al. (2007) \\
\hline IRL & $\begin{array}{l}\text { Monasterevin } \\
\text { bypass }\end{array}$ & 18.430 & 08.2003-12.2005 & 42 & - & 40 & - & - & 150 & 21,8 & - & Desta et al. (2007) \\
\hline NL & $\begin{array}{l}\text { Motorway A1, } \\
\text { Laren }\end{array}$ & - & $01.2003-09.2004$ & 80 & 4,1 & 117 & 3,8 & 29 & 290 & 2,36 & - & Tromp et al. (2012) \\
\hline NL & $\begin{array}{l}\text { A7, Amsterdam (im- } \\
\text { pervious asphalt) }\end{array}$ & 53.000 & 07.1994-09.1195 & $3-6$ & 5 & 121 & 5 & 93 & 452 & 5,2 & 4 & Berbee et al. (1999) \\
\hline NL & $\begin{array}{l}\text { A9, Amsterdam } \\
\text { (pervious asphalt) }\end{array}$ & 83.00 & 07.1994-09.1195 & $3-6$ & 1 & 40 & 1 & 7 & 47 & 0,3 & $<0,1$ & Berbee et al. (1999) \\
\hline JP & Autobahn, Osaka & 75.000 & 08.1997-11.1997 & 4 & 6,5 & 66 & 5,5 & 34 & 648 & 1,28 & - & Shinya et al. (2000) \\
\hline JP & Autobahn, Osaka & 62.000 & 05.1999-08.2000 & 8 & - & 68 & - & 31 & 713 & 0,69 & - & Shinya et al. (2003) \\
\hline AUS & $\begin{array}{l}\text { Parramatta Road, } \\
\text { Sydney }\end{array}$ & 84.500 & $08.2007-04.2008$ & 8 & - & 105 & - & 47 & 348 & - & - & Davis and Birch (2010) \\
\hline \multicolumn{3}{|c|}{ Mittelwert von allen Messungen } & & & 13,1 & 86 & 13,5 & 59 & 383 & 4,8 & 1,9 & - \\
\hline \multicolumn{3}{|c|}{ Mittelwert ohne Extremwerte } & & & 8,8 & 76 & 11,1 & 40,8 & 279 & 2,1 & 1,5 & - \\
\hline
\end{tabular}

und der maximalen stündlichen Niederschlagsintensität. Atmosphärische Ablagerungen, Probenahmestrategien und Probeanzahl pro Regenereignis sind weitere mögliche potenzielle Einflussfaktoren (Crabtree et al. 2008; Helmreich et al. 2010; Kayhanian et al. 2012). So konnte mit den verfügbaren Literaturdaten kein zusätzlicher statistischer Zusammenhang in Hinblick auf JDTV allein ermittelt werden.
Spurenstoffe in Niederschlagsabflüssen von Verkehrsflächen sind sowohl in gelöster als auch in partikulärer Phase präsent. Die Verteilung der Schwermetalle zwischen der gelösten und der partikulären Phase ist besonders für die Beurteilung der Toxizität des Niederschlagsabflusses sowie für die Entwicklung oder Auswahl von Behandlungssystemen entscheidend (Furumai et al. 2002; Haile et al. 2016; Helm- reich et al. 2010; Kayhanian et al. 2012). Trotz einiger Inkonsistenzen in den Literaturdaten zeigen die vorhandenen Monitoringdaten, dass die größten Fraktionen an Schwermetallen $(\mathrm{Cr}, \mathrm{Cu}$, $\mathrm{Ni}, \mathrm{Pb} \& \mathrm{Zn}$ ) und PAK in Verkehrsabflüssen in der Regel überwiegend in der partikulären Phase liegen. Somit liegen die partikulären Anteile für die Schwermetalle (Cr, $\mathrm{Cu}, \mathrm{Ni}$ und $\mathrm{Zn})$ bei jeweils über $50 \%$ und für $\mathrm{Pb}$ bei über 

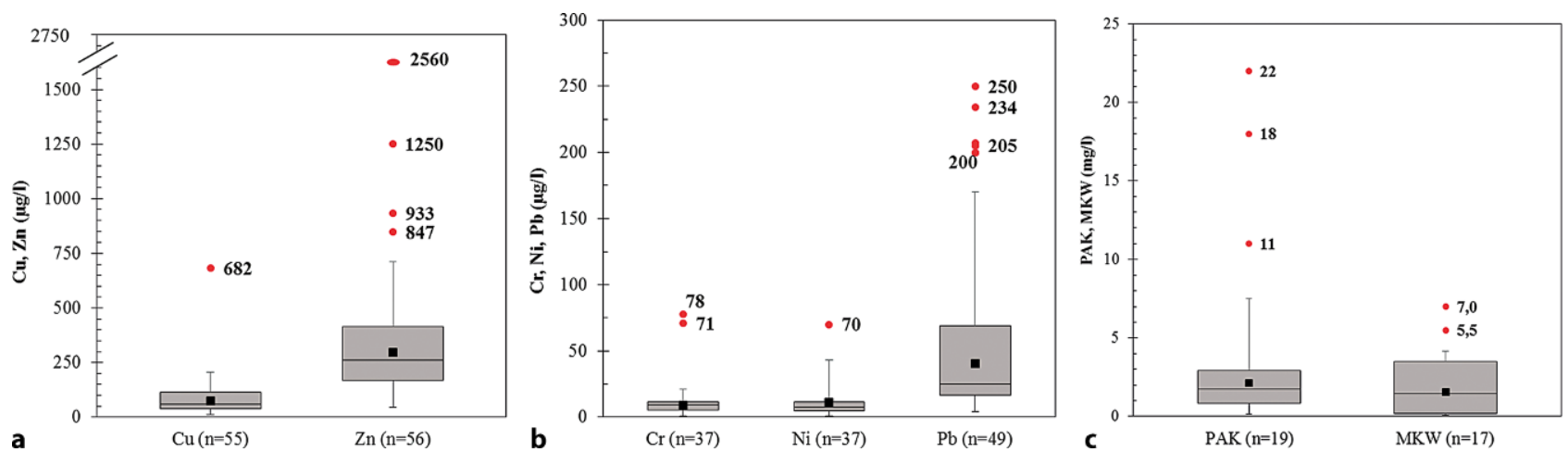

Abb. 1 Schwermetall-, PAK- und MKW-Konzentrationen (gesamt) in Niederschlagsabflüssen von stark befahrenen Straßen mit JDTV > 15.000 Fahrzeuge pro Tag (Zusammenstellung der Tab. 1). Box-Whisker-Plots: oberes bzw. unteres Ende der Box entspricht dem oberen bzw. unteren Quartil, der Strich in der Box dem Median und das kleine Quadrat (schwarz) in der Box der mittleren Konzentration; die kleinen Kreise (rot) stellen Extremwerte dar

Tab. 3 Überblick über die Prüfmethoden und Kriterien für Niederschlagsbehandlungssysteme für den Abfluss von Verkehrsflächen

\begin{tabular}{|c|c|c|c|c|}
\hline Literatur & Land & Prüfmethode & Prüfsubstanz ${ }^{* *}$ & Einleitung in \\
\hline ÖNORM B 2506-3 (2016) & AUT & Labor & AFS, Cu, Zn, NaCl, MKW & Grundwasser \\
\hline ÖNORM B 2506-2 (2012) & AUT & Keine Angabe & AFS, Cu, Zn, MKW & Grundwasser \\
\hline ASTRA (2016a, 2016b) & $\mathrm{CH}$ & Anlage & AFS, $\mathrm{Cu}, \mathrm{Zn}$ & Keine Angabe \\
\hline ASTRA (2010) & $\mathrm{CH}$ & Anlage & AFS, Cu, Zn, PAK, DOC & Einleitung in die Gewässer \\
\hline Schmidt et al. (2015) & $\mathrm{CH}$ & Labor \& Anlage & $\begin{array}{l}\text { Cu, Zn, Pestizide (z. B. Meco- } \\
\text { prop Diuron) }\end{array}$ & Keine Angabe \\
\hline DIBt $(2011,2012)$ & DEU & Labor \& Pilotaanlage & AFS, Cu, Zn, NaCl, MKW & Grundwasser \\
\hline LANUV (2014) & DEU & Labor oder Anlage & AFS, Cu, Zn, NaCl, MKW & Oberflächengewässer \\
\hline NJDEP (2009) & USA, NJ & Anlage & AFS & Keine Angabe \\
\hline NJDEP $(2013 ; 2017)$ & USA, NJ & Labor & AFS & Keine Angabe \\
\hline Sample et al. (2012) & USA, VA & Labor oder Anlage & AFS, $\mathrm{P}$ & Keine Angabe \\
\hline WDOE (2011) & USA, WA & Labor \& Anlage & AFS, Cu, Zn, MKW, P & Grundwasser- und Oberflächengewässer \\
\hline Boogaard (2015) & NL & Labor \& Anlage & AFS & Grundwasser- und Oberflächengewässer \\
\hline Victorian Stormwater Committee (2006) & AUS & Keine Angabe & AFS, gelösten Stoffe, TN, TP & Keine Angabe \\
\hline ARC (2003) & NZL & Anlage & AFS & Keine Angabe \\
\hline Fassman (2012) & NZL & Anlage & AFS, Cu, Zn & Meerwasser \\
\hline Monrabal-Martinez et al. (2017) & NOR & Labor & $\mathrm{Cu}, \mathrm{Ni}, \mathrm{Pb}, \mathrm{Zn}$ & $\begin{array}{l}\text { Mäßig verschmutzte Oberflächengewäs- } \\
\text { ser }\end{array}$ \\
\hline
\end{tabular}

$80 \%$ (Ball et al. 1998; Helmreich et al. 2010; Huber et al. 2016; Kayhanian et al. 2012; Sansalone und Buchberger 1997).

\section{4 Überblick über bestehende Prüfmethoden}

Überblick über die internationale Behandlung von Niederschlagsabflüssen von Verkehrsflächen, Prüfmethoden und vorgeschlagene Prüfsubstanzen (Tab. 3).

\section{Prüfverfahren nach ÖNORM B 2506-3}

In Österreich wurde eine Prüfmethode entwickelt, um die Eignung von techni- schen Filtermaterialien für die Regenwasser-Sickeranlagen für Abläufe von Dachflächen und befestigten Flächen zu überprüfen (ÖNORM B 2506-3 2016). Die Laborprüfmethode wird verwendet, um die Entfernung von Partikeln (abfiltrierbare Stoffe AFS), gelösten Schwermetallen (Blei, Kupfer und Zink) und Mineralölkohlenwasserstoffen $\mathrm{zu}$ beschreiben und die Remobilisierung der Schwermetalle durch Streusalz $(\mathrm{NaCl})$ zu prüfen. Zusätzlich werden auch die Durchlässigkeit des Filtermaterials und seine Veränderung durch Partikelzugabe überprüft.

Die Prüfmethode besteht aus acht Teilprüfungen, die in der angegebenen Reihenfolge durchgeführt werden:
1. Infiltrationsrate und Suffusionsstabilitätstest

2. Partikelretention I

3. Schwermetallrückhalt $(\mathrm{Cu}, \mathrm{Pb}$ und $\mathrm{Zn}$ )

3.1 Versuche im Überstaubetrieb 3.2 Kapazitätsprüfung mit 4 Jahresfracht

4. Mineralölrückhalt

5. Partikelretention II

6. Bestimmung der Änderung der Infiltrationsrate und der Remobilisierung der AFS

7. Remobilisierung von Schwermetallen durch Beschickung mit $5 \mathrm{~g} / \mathrm{L}$ $\mathrm{NaCl}$

8. Säureneutralisationskapazität 
Tab. 4 Ausgewählte Konzentrationen der Niederschlagswässer von befestigten Flächen für die Berechnung der Jahresfrachten

\begin{tabular}{|c|c|c|c|c|c|c|}
\hline Parameter & $\begin{array}{l}\text { AFS } \\
\text { (mg/L) }\end{array}$ & $\begin{array}{l}\mathrm{Pb} \\
(\mu \mathrm{g} / \mathrm{L})\end{array}$ & $\begin{array}{l}\mathrm{Cu} \\
(\mu \mathrm{g} / \mathrm{L})\end{array}$ & $\begin{array}{l}\mathrm{Zn} \\
(\mu \mathrm{g} / \mathrm{L})\end{array}$ & $\begin{array}{l}\text { Mineralöl } \\
\text { (mg/L) }\end{array}$ & $\begin{array}{l}\mathrm{NaCl} \\
(\mathrm{mg} / \mathrm{L})\end{array}$ \\
\hline Prüfung im Überstaubetrieb & 90 & 50 & 100 & 400 & 5,0 & - \\
\hline Kapazitätsprüfung & - & 200 & 400 & 1600 & - & - \\
\hline Remobilisierung von Schwermetallen & - & - & - & - & - & 5000 \\
\hline
\end{tabular}

Bei der Prüfung wird das Filtermaterial mit vier Jahresfrachten für Schwermetalle bzw. AFS und einer Jahresfracht Mineralöl beschickt. Zur Berechnung der Jahresfrachten wurde eine mittlere Belastung des Niederschlagsabflusses von befestigten Flächen mit Partikeln, Schwermetallen und Mineralöl der Literatur entnommen und die in Tab. 4 angegebenen Konzentrationen ausgewählt.

Die Prüfung gilt als bestanden, wenn ein definierter Mindestrückhalt bzw. eine definierte Konzentration eingehalten wird.

Die Prüfungen werden für unterschiedliche hydraulische Belastungen (Flächenverhältnisse (As: Ared) von 1:15 bis 1:250) definiert. Bei der Prüfung von Anlagen mit Flächenverhältnissen (As: Ared) größer 1:100 ist eine Absetzanlage erforderlich, die zumindest $50 \%$ der Partikel entfernt.

6 Anwendung der ÖNORM B 25063 für die Eignungsprüfung von Substraten zur Reinigung von Niederschlagsabflüssen vom hochrangigen Straßennetz

Da die ÖNORM B 2506-3 als Grundlage für die ÖNORM B 2506-1 und -2 und für das ÖWAV-Regelblatt 45 erstellt wurde und der Geltungsbereich dieser ÖNORM Einzugsflächen für die Versickerung von Abflüssen von Dachflächen, befestigten Bodenflächen, wie z. B. Höfen, Zufahrten, Gehwegen, Terrassen, Pkw-Abstellflächen, Lager- und Ladeflächen sowie Verkehrsflächen bis $\mathrm{zu}$ einer Belastung von 5000 JDTV (durchschnittliche tägliche Verkehrsstärke), nicht aber den Abfluss von übergeordneten Verkehrsflächen wie z. B. Autobahnen oder Hauptverkehrsstraßen umfasst, und auch das ÖWAVRegelblatt 45 für JDTV $>15.000 \mathrm{Kfz} / 24 \mathrm{~h}$ auf die RVS verweist, stellt sich die Frage, ob die ÖNORM B 2506-3 für die Versickerung von Abwasser von hochrangigen Straßen anwendbar ist.

Dazu ist festzustellen, dass die Schwermetallbelastungen nicht nur von der Verkehrsbelastung abhängen und auch innerhalb vergleichbarer JDTV sehr unterschiedlich sein können (Tab. 2). Die in der Prüfmethode der ÖNORM B 2506-3 verwendeten Schwermetallkonzentrationen und auch die MKW-Konzentrationen sind höher als die mittleren Konzentrationsniveaus von hochrangigen Straßen. Zusätzlich werden die Prüfungen mit gelösten Schwermetallen bei niedrigem pH-Wert ( $\mathrm{pH} 5,5$ bzw. 5,8) durchgeführt. Dies bietet eine zusätzliche Sicherheit, da im Straßenabwasser ein erheblicher Teil der Schwermetalle an Partikel gebunden ist und mit diesen entfernt wird. Für die Mineralölprüfung wird Heizöl EL direkt auf die Oberfläche aufgetragen, nach dem Eindringen des Heizöls wird die Säule im Überstaubetrieb beschickt. Für die Prüfung des Partikelrückhalts und der Suffusionsneigung der Materialien werden sehr feine Quarzpartikel ( $>50$ Gew-\% $<63 \mu \mathrm{m}$ ) verwendet, die weder Mineralöle noch Schwermetalle adsorbieren. All dies stellt eine Verschärfung der Konditionen gegenüber der Praxis dar (Haile und Fürhacker 2015; Haile et al. 2016).

Aus den in der ÖNORM B 2506-3 gewählten Prüfbedingungen und Prüfkriterien für die Prüfung der technischen Filtermaterialien nach ÖNORM B 2506-2 und ÖWAV-RB 45 ergibt sich, dass die erfolgreich geprüften Materialien aus wissenschaftlicher Sicht geeignet sind, auch die Anforderungen an die Reinigung von Straßenabwässern von hochbelasteten Straßen mit hohen JDTV, z.B. des hochrangigen
Straßennetzes, zu erfüllen. Es sei noch darauf hingewiesen, dass die Prüfung der technischen Filtermaterialien nach ÖNORM B 2506-3 für die Versickerung in das Grundwasser erstellt wurde.

Erfahrungen aus durchgeführten Projekten am Institut für Siedlungswasserbau der Universität für Bodenkultur Wien zeigen, dass technische Filtermaterialien eine gute Reinigungsleistung für die Behandlung von Spurenstoffen von Straßenabwässern aufweisen.

\section{Schlussfolgerungen}

Auch wenn die ÖNORM B 2506-3 als Grundlage für die ÖNORM B 2506-1 und -2 und für das ÖWAV-Regelblatt 45 (Anwendungsbereiche JDTV $<5000 \mathrm{bzw}$. $<15.000 \mathrm{Kfz} / 24 \mathrm{~h}$ ) erstellt wurde, ergibt sich, dass aufgrund der in der ÖNORM B 2506-3 gewählten Prüfbedingungen und Prüfkriterien aus wissenschaftlicher Sicht auch die Anforderungen der Reinigung von Straßenabwässer von hochbelasteten Straßen mit hohen JDTV, wie jener des hochrangigen Straßennetzes, erfüllt werden.

Acknowledgements Open access funding provided by University of Natural Resources and Life Sciences Vienna (BOKU).

Open Access Dieser Artikel wird unter der Creative Commons Namensnennung 4.0 International Lizenz (http:// creativecommons.org/licenses/by/4. $0 /$ deed.de) veröffentlicht, welche die Nutzung, Vervielfältigung, Bearbeitung, Verbreitung und Wiedergabe in jeglichem Medium und Format erlaubt, sofern Sie den/die ursprünglichen $\mathrm{Au}$ tor(en) und die Quelle ordnungsgemäß nennen, einen Link zur Creative Commons Lizenz beifügen und angeben, $\mathrm{ob}$ Änderungen vorgenommen wurden. 
ARC (2003): Stormwater Management Devices: Design Guideline Manual. Technical Publication 10. Auckland, Auckland Regional Council, SS $1-249$.

ASTRA (2016a): Technisches Merkblatt Bauteil Entwässerung und Strassenabwasserbehandlung: Leistungsprüfung neuer Verfahren, 21001 10468. Fachhandbuch T/U (Trassee/Umwelt), Abteilung Strasseninfrastruktur I, Bundesamt für Strassen ASTRA, SS, 1-8.

ASTRA (2016b): Technisches Merkblatt Bauteil, Entwässerung und Strassenabwasserbehandlung: Funktionsprüfung bei der Abnahme und bei der periodischen Kontrolle, 21 001-10469. Fachhandbuch T/U (Trassee/Umwelt), Abteilung Strasseninfrastruktur I, Bundesamt für Strassen ASTRA, SS, 1-10.

ASTRA (2010): Straßenabwasserbehandlungsverfahren, Stand der Technik. Bundesamt für Straßen ASTRA. Bundesamt für Umwelt BAFU, Bern, SS. 1-130.

Ball, J.E., Jenks, R., Aubourg, D. (1998): An assessment of the availability of pollutant constituents on road surfaces. Sci. Total Environ. 209(2-3), 243-254.

Barrett, M. E., Irish Jr, L.B., Malina Jr., J.F., Charbeneau, R.J. (1998): Characterization of highway runoff in Austin, Texas, area. J. Environ. Eng. 124 131-137.

Berbee, R., Rijs, G., de Brouwer, R., Van Velzen, l. (1999): Characterization and Treatment of $\mathrm{Ru}-$ noff from Highways in the Netherlands Paved with Impervious and Pervious Asphalt. Water Environment Research 71 (2), 183-190.

Boogaard, F.C. (2015): Stormwater characteristics and new testing methods for certain sustainable urban drainage systems in The Netherlands. Dissertation, Technische Universiteit Delft. ISBN: 978-94-6259-745-7.

Clara, M., Ertl, T., Giselbrecht, G., Gruber, G. Hofer, T., Humer, F., Kretschmer, F., Kolla, L. Scheffknecht, C., Weiß, S. und Windhofer, G. (2014): Spurenstoffemissionen aus Siedlungsgebieten und von Verkehrsflächen. Studie im Auftrag des Bundesministeriums für Land- und Forstwirtschaft, Umwelt und Wasserwirtschaft. Wien, Österreich, SS. 1-1354.

Crabtree, B., Dempsey, P., Johnson, I., Whitehead, M. (2008): The development of a riskbased approach to managing the ecological impact of pollutants in highway runoff. Water Sci. Technol. $57,1595-1600$.

Davis, B. and Birch, G. (2010): Comparison of heavy metal loads in stormwater runoff from major and minor urban roads using pollutant yield rating curves. Environmental Pollution 158 (8), 2541-2545.

Davis, A.P., Shokouhian, M., Ni, S. (2001): Loading estimates of lead, copper, cadmium, and zinc in urban runoff from specific sources. Chemosphere 44, 997-1009.

Desta, M.B., Bruen, M., Higgins, N., Johnston, P (2007): Highway runoff quality in Ireland. Journal of Environmental Monitoring 9(4), 366-371. DIBt (2011): Zulassungsgrundsätze für Niederschlagswasserbehandlungsanlagen. Teil 1: Anlagen zum Anschluss von Kfz-Verkehrsflächen bis $2.000 \mathrm{~m}^{2}$ und Behandlung des Abwassers zur anschließenden Versickerung in Boden und Grundwasser. Deutsches Institut für Bautechnik, Berlin, Entwurf Februar 2011, SS. 1-20.

DIBt (2012): Zulassungsgrundsätze Niederschlagswasserbehandlungsanlagen; Teil 2: Wasserdurchlässige Beläge für Kfz-Verkehrsflächen für die Behandlung des Abwassers zur anschließenden Versickerung in Boden und Grund- wasser (Abwasserbehandelnde Flächenbeläge) Deutsches Institut für Bautechnik, Berlin.

Dierkes, C., Geiger, W.F. (1999): Pollution retention capabilities of roadside soils. Water Sci. Technol. 39 (2), 201-208.

Dobner und Holthuis (2011): Praxiserprobung und technische Optimierung eines neuartigen Hochleistungs-Pflanzenfilterverfahrens zur Behandlung belasteter Niederschlagswässer. AiFVorhaben-Nr: 15508 N/1 und N/2 Gemeinsame Abschlussbericht für den Zeitraum: 01.02.2008 bis 30.11.2010. SS. 1-172.

Driscoll, E.D., Shelley. P.E. and Strecker. E.W. (1990): Pollutant Loadings and Impacts from Highway Stormwater Runoff. Vol. I: Design Procedure. Technical Report No. FHWA-RD-88-07. prepared for the Federal Highway Administration. Washington. D.C.

Fassman, E. (2012): Stormwater BMP treatment performance variability for sediment and heavy metals. Separation and Purification Technology $84,95-103$.

Fuerhacker, M., Haile, T.M., Monai, B., Mentler, A. (2011): Performance of a filtration system equipped with filter media for parking lot runoff treatment. Desalination 275, 118-125.

Fürhacker, M., Haile, T.M., Schärfinger, B., Kammerer, G., Allabashi, R., Magnat, S. (2013): Entwicklung von Methoden zur Prüfung der Eignung von Substraten für die Oberflächenwasserbehandlung von Dach- und Verkehrsflächen. Fördervertrag GZ B100121. Wien. Österreich, SS. $1-240$.

Furumai, H., Balmer, H., Boller, M. (2002): Dynamic Behavior of Suspended Pollutants and Particle Size Distribution in Highway Runoff. Water Sci. Technol. 46 (11-12), 413-418.

Geiger-Kaiser, M., \& Jäger, P. (2005): Reinigung von Straßenabwässern. Wirksamkeit von Retentionsfilterbecken zur Reinigung von Straßenoberflächenwässern. Gewässerschutz 11, SS $1-54$.

Gill, L.W., Ring, P., Casey, B., Higgins, N.M.P., Johnston, P.M. (2017): Long term heavy metal removal by a constructed wetland treating rainfall runoff from a motorway. Science of the Total Environment 601-602, 32-44.

Göbel, P., Dierkes, C., Coldwey, W.G. (2007): Stormwater runoff concentration matrix for urban areas. Journal of Contaminant Hydrology Vol. 91, 26-42.

Grotehusmann, D. und Kasting, U.(2009): Vergleich der Reinigungsleistung von Retentionsbodenfiltern und Versickerungsanlagen an Bundesfernstraßen. Schlussbericht zum Forschungsprojekt FE-Nr. 05.141/2005/GRB im Auftrag der Bundesanstalt für Straßenwesen (BASt), SS. 1-53.

Haile, T.M., Fürhacker, M. (2015): Prüfmethode zur Filterwirkung und -eignung. Versickerung von Niederschlagswässern: ÖWAV-Regelblatt 45 Rahmenbedingungen, Bemessung und Betrieb von Versickerungsanlagen, 06.11.2015, Wien. Österreichische Wasser- und Abfallwirtschaftsverband.

Haile, T.M., Hobiger, G., Kammerer, G., Allabashi, R., Schaerfinger, B., Fuerhacker, M. (2016): Hydraulic Performance and Pollutant Concentration Profile in a Stormwater Runoff Filtration Systems. WATER AIR SOIL POLL. 227(1)

Helmreich, B., Hilliges, R., Schriewer, A., Horn H. (2010): Runoff pollutants of a highly trafficked urban road e correlation analysis and seasona influences. Chemosphere 80, 991-997.

Herrera Environmental Consultants (2007): Untreated Highway Runoff in Western Washington. White Paper
Hilliges, R., Schriewer, A., Helmreich, B. (2013): A three-stage treatment system for highly polluted urban road runoff. Journal of Environmental Management 128, 306-312.

Höfler, M., Weidinger, A., Buschbeck, G., Eder, J. (2004): Bericht Projekt Regenbecken. Autobahnregenbecken Mönchsgraben. Endbericht. Land Oberösterreich, Abteilung Wasserwirtschaft Gewässerschutz, Linz.

Horstmeyer, N., und Helmreich, B. (2014): Erfahrung mit Schwermetallbelastungen beim Betrieb von Versickerungsmulden. Zukunftsfähige Bewirtschaftungskonzepte für Niederschlagswasser. 42. Abwassertechnisches Seminar, Nr. 211, 129-144. prepared for Washington State Department of Transportation, Seattle May 2007.

Huber, M., Welker, A., Helmreich, B. (2015): Critical review of heavy metal pollution of traffic area runoff: Occurrence, influencing factors, and partitioning. Science of the Total Environment 541, 895-919

Hürlimann, J. (2011): Auswirkungen von Straßenabwasser aus Oberflächengewässer. Gewässerökologische Beurteilung: Abwasser Hauptartikel, 793-801.

Kayhanian, M., Singh, A., Suverkropp, C., Borroum, S. (2003): Impact of annual average daily traffic on highway runoff pollutant concentrations. Journal of Environmental Engineering 129 (11), 975.

Kayhanian, M., C. Suverkropp, A. Ruby and K. Tsay. (2007): Characterization and prediction of highway runoff constituent event mean concentration. Journal of environmental management. 85, 279-295.

Kayhanian, M., McKenzie, E.R., Leatherbarrow, J. E., Young, T.M. (2012): Characteristics of road sediment fractionated particles captured from paved surfaces, surface run-off and detention basins. Science of the Total Environment, 439, 172-186.

Kocher, B., Brose, S., Chlubek, A., Karagüzel, N., Klein, N., Siebertz, I. (2010): Stoffeintrag in Straßenrandböden: Messzeitraum 2005/2006 Berichte der Bundesanstalt für Straßenwesen, Bergisch Gladbach, SS. 1-45.

Langbein, S., Steiner, M., Boller, M. (2006): Schadstoffe im Straßenabwasser einer stark befahrenen Straße und deren Retention mit neuartigen Filterpaketen aus geotextil und Adsorbermaterial. Schlussbericht des Forschungsprojekts, SS. 1-130.

LANUV (2014): Entwicklung einer Prüfvorschrift für dezentrale Behandlungsanlagen für Verkehrsflächenabflüsse bei Einleitung in Oberflächengewässer. Im Auftrag des Ministeriums für Klimaschutz, Umwelt, Landwirtschaft, Naturund Verbraucherschutz des Landes NordrheinWestfalen. https://www.lanuv.nrw.de/uploads tx mmkresearchprojects/Bericht_Welker 01 2014.pdf. Zugegriffen: 13.06.2017.

Lau, S.-L., Han, Y., Kang, J.-H., Kayhanian, M., Stenstrom, M.K. (2009): Characteristics of highway stormwater runoff in Los Angeles: metals and polycyclic aromatic hydrocarbons. Water Environ. Res. 81, 308-318.

Legret, M. and Pagotto, C. (1999): Evaluation of pollutant loadings in the runoff waters from a major rural highway. Sci. Total Environ. 235 (1-3), 143-150.

McKenzie, E.R., Money, J.E., Green, P.J., Young, T.M. (2009): Metals associated with stormwaterrelevant brake and tire samples. Sci. Total Environ. 407(22), 5855-5860.

Monrabal-Martinez, C., Ilyas, A., Muthanna, T.M. (2017): Pilot Scale Testing of Adsorbent 
Amended Filters under High Hydraulic Loads for Highway Runoff in Cold Climates. Water 9 (230), doi:10.3390/w9030230.

Moy, F., Crabtree, R., Simms, T. (2003) Long term monitoring of pollution from highway runoff. Environment Agency R\&D Report No. P2-038. Seiten, 1-172. https://www.gov.uk/government/ uploads/system/uploads/attachment_data/ file/290643/sp2-038-tr1-e-e.pdf. Zugegriffen: 13.06.2017.

NJDEP (2009): Protocol for Total Suspended Solids Removal Based on Field Testing: Amendments to TARP Protocol Dated August 5, 2009 Revised December 15, 2009. Trenton, NJ: New Jersey Department of Environmental Protection. http://www.state.nj.us/dep/stormwater/ pdf/field_protocol_12_15_09.pdf. Zugegriffen: 18.07.2017.

NJDEP (2013): Procedure for Obtaining Verification of a Stormwater Manufactured Treatment Device from New Jersey Corporation for Advanced Technology. http://www.njstormwater. org/pdf/njcat-mtd-process-1-25-13.pdf. Zugegriffen: 18.07.2017.

NJDEP (2017): Laboratory Test Protocols and Verification Procedure: New Jersey Corporation for Advanced Technology Interpretations. http://www.njcat.org/uploads/docs/ NJCATInterpretations-LabTestProtocols\%20June \%202017.pdf. Zugegriffen: 18.07.2017.

ÖWAV-RB 45 (2015): Oberflächenentwässerung durch Versickerung in den Untergrund. Österreichischer Wasser- und Abfalwirtschaftverband Austrian Standards plus Publishing 1020 Wien. ÖNORM B 2506-2 (2012): Regenwasser-Sickeranlagen für Abläufe von Dachflächen und befestigten Flächen - Teil 2: Qualitative Anforderungen an das zu versickernde Regenwasser sowie Anforderungen an Bemessung, Bau und Betrieb von Reinigungsanlagen. Österreichisches Normungsinstitut.

ÖNORM B2506-3 (2016): Regenwasser-Sickeranlagen für Abläufe von Dachflächen und befestigten Flächen - Teil 3: Filtermaterialien Anfor- derungen und Prüfmethoden. Österreichisches Normungsinstitut.

Sample, D.J., Grizzard, T.J., Sansalone, J., Davis, A.P., Roseen, R.M., Walker, J. (2012): Assessing performance of manufactured treatment devices for the removal of phosphorus from urban stormwater. J. Environ. Manag. 113, 279-291 Sansalone, J.J. \& Buchberger, S.G. (1997): Partitioning and First Flush of Metals in Urban Roadway Storm Water. J. Environ. Eng.. 123 (2), 134-143.

Scheffknecht, C., \& Prodinger, H. (2007): Abwasser- und Bodenuntersuchungen am Retentionsfilterbecken Landesstraße L 202 HardBregenz (Emissionsmessstelle Bregenzerachbrücke). Umweltinstitut Vorarlberg, pp 1-21. http://www.vorarlberg.at/pdf/filterbecken 1202.pdf. Zugegriffen: 25.01.2017

Scheiwiller. E., Ochsenbein, U., Kaufmann, P., Rudin, M. (2008): Schadstoffabschwemmungen: Am Beispiel von Hochleistungsstraßen. gwa. SS 539-546.

Schmidt, S., Burkhardt, M., Gohl, M., Boller, M. (2015): Neue Konzepte zur dezentralen Behandlung von Regenwasserabflüssen. Aqua Urbanica 2015, Stuttgart, 07-08 October 2015. Stuttgarter Berichte zur Siedlungswasserwirtschaft, Vol. 225. pp. 85-90

Schütte, M. (1997): Messungen der Regenwasserverschmutzung im Einzugsgebiet des Biesdorfer Baggersees. Ingenieurgesellschaft für Stadthydrologie, Hannover. Zitiert in Huber et al. (2016).

Shinya, M., Tsuchinaga, T, Kitano, M., Tamada., Y., Ishikawa, M. (2000): Characterization of Heavy Metals and Polycyclic Aromatic Hydrocarbons in Urban Highway Runoff. Water Sci. Technol. 42 (7-8), 201-208.

Shinya, M., Tsuruho, K., Konishi, T., Ishikawa M. (2003): Evaluation of factors influencing diffusion of pollutant loads in urban highway runoff. Water Science and Technology 47, 227-232. Steiner, M., Langbein, S., Boller, M. (2006): Bankette bestehendee Straßen - Untersuchung der Versickerung von Strassenabwasser über Stras- senrandstreifen an einer bestehenden Strasse. Schlussbericht des Forschungsprojekts, SS. 1-59. Steiner, M., Goosse. P., Hermann, E., Boller, M. (2008): Leistungsbeurteilung der SABA Attinghausen. Halbzeit des Monitorings. GWA 88.7, 531-538.

Stotz, G. (1987): Investigations of the properties of the surface water runoff from federal highways in the FRG. Sci. of the Total Envir. 59: 329-337.

Thomson, N.R., McBean, E.A., Snodgrass, W. (1997): Highway stormwater runoff quality: development of surrogate parameter relationships. Water Air Soil Pollut. 94, 307-347.

Thorpe, A. and Harrison, R.M. (2008): Sources and properties of non-exhaust particulate matter from road traffic: a review. Sci. Total Environ. 400 (1-3), 270-282.

Tromp, K., Lima, A.T., Barendregt, A., Verhoeven, JT A (2012): Retention of heavy metals and poly-aromatic hydrocarbons from road water in a constructed wetland and the effect of deicing. Journal of Hazardous Materials 203-204, 290-298.

Victorian Stormwater Committee (2006): Urban Stormwater: Best Practice Environmenta Management Guidelines. Commonwealth Scientific and Industrial Research Organisation, pp. $1-268$.

WDOE (2011): Technical Guidance Manual for Evaluating Emerging Stormwater Treatment Technologies: Technology Assessment Protocol - Ecology (TAPE), Publication No. 11-10-061, 2011 ed. Washington State Department of Ecology, Lacey, WA, SS. 1-73. https://fortress.wa gov/ecy/publications/summarypages/1110061. html. Zugegriffen: 19.07.2017.

Zafra, C.A., Temprano, J., Tejero, I. (2011): Distribution of the concentration of heavy metals associated with the sediment particles accumulated on road surfaces. Environmental Technology 32(9), 997-1008.

Zbinden, R., Goossse, P., Steiner, M. (2015): Strassen und Verkehr. VSS: Fachartikel, 33-38. 